Editor's Note: These short reviews of a recent paper in the Journal, written exclusively by graduate students or postdoctoral fellows, are intended to mimic the journal clubs that exist in your own departments or institutions. For more information on the format and purpose of the Journal Club, please see http://www.jneurosci.org/misc/ifa_features.shtml.

\title{
Listen to Your Visual Cortex: Effects of Occipital Transcranial Magnetic Stimulation on Auditory Stimulus Detection
}

\author{
Oliver Doehrmann \\ Institute of Medical Psychology, Johann Wolfgang Goethe-University, D-60528 Frankfurt am Main, Germany \\ Review of Romei et al. (http://www.jneurosci.org/cgi/content/full/27/43/11465)
}

Despite the apparently strong dominance of visual perception, we usually experience our environment through simultaneous stimulation of more than one sensory channel. For instance, watching a movie is in most cases a visual and auditory experience. This integration of information from different sensory modalities can bring about behavioral advantages, for example concerning stimulus detection and discrimination in noisy environments.

Although many questions about the neural underpinnings of processing within single sensory domains have been tackled successfully over several decades of neuroscientific research, related questions of how and where in the brain these different processing streams are integrated have begun to be addressed only more recently. Interestingly, in nonhuman primates, integrative processing caused by the presentation of stimuli in more than one sensory modality has recently been reported in regions as early as primary auditory or visual cortex (Lakatos et al., 2007). Thus, this active field of research might call into question traditional views of brain organization that

Received Nov. 16, 2007; revised Nov. 30, 2007; accepted Nov. 30, 2007. Correspondence should be addressed to Oliver Doehrmann at the above address. E-mail: doehrmann@med.uni-frankfurt.de.

DOI:10.1523/JNEUROSCI.5101-07.2008

Copyright $\odot 2008$ Society for Neuroscience $\quad$ 0270-6474/08/280001-02\$15.00/0 postulate strict parceling into unisensory and multisensory cortical levels (Ghazanfar and Schroeder, 2006).

A recently published study by Romei et al. (2007) offers valuable additional insights into early auditory-visual (AV) integration effects in human subjects. Their subjects were instructed to detect simple stimuli (a briefly presented pure tone, a small white disk, or a combination of the two), and their reaction times (RTs) were measured. In some cases, these three experimental conditions were accompanied by single-pulse transcranial magnetic stimulation (TMS), either over the occipital poles of the subjects' heads or over a control site at the vertex. The rationale for this is that TMS generates short electromagnetic pulses that transgress the skull and stimulate the underlying cortical tissue, facilitating or suppressing a subject's task performance, depending on stimulation site, intensity, and timing. Additionally, TMS stimulation can be restricted to specific time intervals after stimulus presentation, thereby offering temporal information in the millisecond range about cortical processing stages. More specifically, TMS effects over visual cortex in a timeframe from 60 to $75 \mathrm{~ms}$ after sensory stimulus onset would suggest an interaction with feedforward processes, whereas later effects might be caused by feedback from higher cortical regions. Thus, varying the delay from 30 to $150 \mathrm{~ms}$ between
TMS and the preceding sensory stimulation in different sensory modalities enabled Romei et al. (2007) to determine the processing type (feedforward or feedback), as well as the critical timeframe of visual cortex involvement in stimulus processing.

In agreement with previous studies, RTs for the detection task without concurrent TMS stimulation [Romei et al. (2007), their Fig. 1 (http://www. jneurosci.org/cgi/content/full/27/43/ $11465 / \mathrm{F} 1)]$ were significantly better for the AV condition than for both unimodal conditions, suggesting a typical behavioral facilitation effect for stimuli presented simultaneously in both modalities. Furthermore, the authors explicitly tested for violations of the so-called race model, according to which the probability of an assumed facilitation effect for AV stimuli has to exceed the sum of the probabilities for the same RT during both unimodal conditions minus an expression of their joint probability. Romei et al. (2007) were able to demonstrate such a violation of the race model over the fastest $40 \%$ of the RT distribution. This suggested that the RT facilitation for AV stimuli is produced by true nonlinear multisensory interactions instead of a simple probability summation.

Most importantly, to investigate where and when these multisensory interactions occurred, the authors applied occipital 
TMS along with sensory stimulation [Romei et al. (2007), their Fig. 2 (http:// www.jneurosci.org/cgi/content/full/27/ $43 / 11465 / F 2)]$. In a time window of 60-75 ms after stimulus presentation, a TMS pulse impaired RTs for visual stimuli but facilitated RTs for auditory stimuli compared with a control TMS pulse (over the vertex) or to baseline without TMS stimulation. In contrast, no significant differential effects between occipital and vertex TMS were found when the pulse was paired with AV stimuli.

The magnitude of facilitation produced by pairing auditory stimulation with occipital TMS was nearly identical to that produced by the simultaneous presentation of AV stimuli in the absence of TMS. Furthermore, there was a significant correlation in the amount of facilitation produced under the two experimental paradigms across subjects [Romei et al. (2007), their Fig. 3 (http://www.jneurosci. org/cgi/content/full/27/43/11465/F3)]. Thus, a combination of tone presentation and successive occipital TMS on the one hand or AV stimulation on the other hand seem to tap into the same processes on a level as early as primary visual cortex.

The authors added several interesting control experiments and further analyses to strengthen these findings. Of particular importance were measurements of phosphenes, visual sensations induced by magnetic or electrical stimulation of neural tissue. First, Romei et al. (2007) were able to show that their results remained essentially the same even when those subjects who were especially prone to experience phosphenes after occipital TMS stimulation were excluded from the analysis. This ruled out the possibility that the results reflected a special case of $\mathrm{AV}$ integration in which a visual stimulus is produced by TMS rather than entering via the conventional retinal pathway.

Second, seven subjects participated in a follow-up experiment, in which the authors determined in how many trials the subjects experienced visual phosphenes when auditory stimuli were presented with occipital TMS at delays between 30 and $150 \mathrm{~ms}$. Consistent with their results from the main experiment, phosphenes were induced significantly more often when occipital TMS pulses were delivered 75-120 ms after auditory stimulus presentation [Romei et al. (2007), their Fig. 4 (http://www.jneurosci.org/cgi/content/ full/27/43/11465/F4)], suggesting the possibility that an auditory stimulus enhanced the excitability of the visual cortex. These results are in line with a recently published study by Ramos-Estebanez et al. (2007), in which phosphene induction was found to be significantly increased after combined subthreshold somatosensory and occipital TMS stimulation.

The paper by Romei et al. (2007) makes an important contribution to studies concerning early integration effects across sensory modalities in cortical regions previously regarded as strictly unisensory. Most interestingly, they were able to use the strength of single-pulse TMS to provide highly specific temporal information about these interactions. This nicely complements neuroimaging data that have a lower temporal resolution, for instance a recent study demonstrating enhanced responses in primary visual and auditory cortices for simultaneously presented AV stimuli in contrast to temporally incongruent stimuli (Noesselt et al., 2007).

Romei et al. (2007) impressively dem- onstrate that TMS is valuable for studying multisensory integration, by showing that early AV interactions are not functionally silent, but behaviorally relevant. This method will certainly be used in future studies within this research field, for example in paradigms using concurrent TMS stimulation over two cortical regions (see, e.g., Pascual-Leone and Walsh, 2001) to further disentangle the role of feedforward and feedback connections. Possibly, its combination with other neuroimaging techniques will provide the key to a better understanding of the multisensory brain.

\section{References}

Ghazanfar AA, Schroeder CE (2006) Is neocortex essentially multisensory? Trends Cogn Sci 10:278-285.

Lakatos P, Chen C-M, O'Connell MN, Mills A, Schroeder CE (2007) Neuronal oscillations and multisensory Interaction in primary auditory cortex. Neuron 53:279.

Noesselt T, Rieger JW, Schoenfeld MA, Kanowski M, Hinrichs H, Heinze H-J, Driver J (2007) Audiovisual temporal correspondence modulates human multisensory superior temporal sulcus plus primary sensory cortices. J Neurosci 27:11431-11441.

Pascual-Leone A, Walsh V (2001) Fast backprojections from the motion to the primary visual area necessary for visual awareness. Science 292:510-512.

Ramos-Estebanez C, Merabet LB, Machii K, Fregni F, Thut G, Wagner TA, Romei V, Amedi A, Pascual-Leone A (2007) Visual phosphene perception modulated by subthreshold crossmodal sensory stimulation. J Neurosci 27:4178-4181.

Romei V, Murray MM, Merabet LB, Thut G (2007) Occipital transcranial magnetic stimulation has opposing effects on visual and auditory stimulus detection: implications for multisensory interactions. J Neurosci 27:11465-11472. 